\title{
TETRAVALENT $s$-TRANSITIVE GRAPHS OF ORDER TWICE A PRIME POWER
}

\author{
JIN-XIN ZHOU ${ }^{凶}$ and YAN-QUAN FENG
}

(Received 4 August 2008; accepted 16 January 2010)

\author{
Communicated by E. A. O’Brien
}

\begin{abstract}
A graph is $s$-transitive if its automorphism group acts transitively on $s$-arcs but not on $(s+1)$-arcs in the graph. Let $X$ be a connected tetravalent $s$-transitive graph of order twice a prime power. In this paper it is shown that $s=1,2,3$ or 4 . Furthermore, if $s=2$, then $X$ is a normal cover of one of the following graphs: the 4-cube, the complete graph of order 5 , the complete bipartite graph $K_{5,5}$ minus a 1 -factor, or $K_{7,7}$ minus a point-hyperplane incidence graph of the three-dimensional projective geometry $P G(2,2)$; if $s=3$, then $X$ is a normal cover of the complete bipartite graph of order 4 ; if $s=4$, then $X$ is a normal cover of the point-hyperplane incidence graph of the three-dimensional projective geometry $P G(2,3)$. As an application, we classify the tetravalent $s$-transitive graphs of order $2 p^{2}$ for prime $p$.
\end{abstract}

2000 Mathematics subject classification: primary 05C25; secondary 20B25.

Keywords and phrases: $s$-transitive graph, symmetric graph, Cayley graph.

\section{Introduction}

For a finite, simple and undirected graph $X$, we use $V(X), E(X)$ and $\operatorname{Aut}(X)$ to denote its vertex set, edge set and full automorphism group. For $u, v \in V(X),\{u, v\}$ is the edge incident to $u$ and $v$ in $X$, and $X(u)$ is the neighborhood of $u$ in $X$, that is, the set of vertices adjacent to $u$ in $X$. An $s$-arc in a graph is an ordered $(s+1)$-tuple $\left(v_{0}, v_{1}, \ldots, v_{s-1}, v_{s}\right)$ of vertices of the graph such that $v_{i-1}$ is adjacent to $v_{i}$ for $1 \leq i \leq s$, and $v_{i-1} \neq v_{i+1}$ for $1 \leq i \leq s-1$. For a subgroup $G$ of the automorphism group $\operatorname{Aut}(X)$ of a graph $X, X$ is said to be $(G, s)$-arc-transitive or $(G, s)$-regular if $G$ acts transitively or regularly on the set of $s$-arcs of $X$, and $(G, s)$-transitive if $G$ acts transitively on the set of $s$-arcs but not on the set of $(s+1)$-arcs of $X$; in particular,

The authors are grateful for the support of the National Natural Science Foundation of China (10871021, 10901015), the Specialized Research Fund for the Doctoral Program of Higher Education in China (20060004026), and the Science and Technology Foundation of Beijing Jiaotong University (2008RC037).

(C) 2010 Australian Mathematical Publishing Association Inc. 1446-7887/2010 \$16.00 
if $G=\operatorname{Aut}(X)$, then $X$ is simply said to be $s$-arc-transitive, $s$-regular or $s$-transitive, respectively. In particular, 1-arc-transitive means arc-transitive or symmetric. A graph $X$ is edge-transitive if $\operatorname{Aut}(X)$ is transitive on $E(X)$.

Let $X$ be a connected symmetric graph, and let $G \leq \operatorname{Aut}(X)$ be arc-transitive on $X$. For a normal subgroup $N$ of $G$, the quotient graph $X_{N}$ of $X$ relative to the orbit set of $N$ is defined as the graph with vertices the orbits of $N$ on $V(X)$ and with two orbits adjacent if there is an edge in $X$ between vertices lying in these two orbits. Assume further that $X$ is $(G, 2)$-arc-transitive. If $N$ is intransitive then either $X_{N} \cong K_{2}$, or $X_{N}$ and $X$ have the same valency. For the former case, $X_{N}$ is sometimes called a trivial normal quotient; for the latter, $X$ is called a $G$-normal cover of $X_{N}$. In particular, if $G=\operatorname{Aut}(X)$ then $X$ is said to be a normal cover of $X_{N}$. If $X$ has no nontrivial normal quotient with respect to $G, X$ is said to be $G$-basic. An $\operatorname{Aut}(X)$-basic graph is simply called a basic graph. Clearly, $X$ is $G$-basic if and only if each nontrivial normal subgroup of $G$ has at most two orbits on $V(X)$. A general approach to the characterization of 2-arc-transitive graphs is to investigate $G$-basic 2 -arc-transitive graphs and their normal covers (see [24]).

Arc-transitive or $s$-transitive graphs with small valencies have received considerable attention in the literature. For instance, Tutte [26] initiated the investigation of cubic $s$ transitive graphs by proving that there exist no finite $s$-transitive cubic graphs for $s \geq 6$, and there has been much subsequent work in this area (see [5, 7, 9-13, 23]). Gardiner and Praeger [14, 15] generally explored tetravalent symmetric graphs by considering their automorphism groups. A lot of work has been done on tetravalent $s$-transitive Cayley graphs as a part of a more general problem dealing with the investigation of the tetravalent edge-transitive Cayley graphs (see [8, 21], for example). Recently, Li et al. [20] classified all vertex-primitive symmetric graphs of valency 3 or 4 .

There also has been a lot of interest in classifications of $s$-transitive graphs of small valencies with given orders. Let $p$ be a prime. The classification of $s$-transitive graphs of order $n p$ and of valency 3 or 4 can be obtained from [3, 4, 27], where $1 \leq n \leq 3$. Feng et al. [10,12,13] classified cubic $s$-transitive graphs of order $n p$ or $n p^{2}$ with $n=4,6,8$ or 10. In [10], Feng et al. investigated the automorphism groups of cubic $s$-transitive graphs of order $2 p^{n}$ and, as an application, classified the cubic $s$-transitive graphs of order $2 p^{2}$.

In this paper, we aim to study the tetravalent $s$-transitive graphs of order $2 p^{n}$. Let $X$ be a connected tetravalent $s$-transitive graph of order $2 p^{n}$. It is shown that $s=1,2,3$ or 4 . Furthermore, if $s=2$, then $X$ is a normal cover of one of the following graphs: $Q_{4}$ (the 4-cube), $K_{5}$ (the complete graph of order 5), $K_{5,5}-5 K_{2}$ (the complete bipartite graph $K_{5,5}$ minus a 1 -factor), or $B^{\prime}(P G(2,2))\left(K_{7,7}\right.$ minus the point-hyperplane incidence graph of a three-dimensional projective geometry $P G(2,2)$ ); if $s=3$, then $X$ is a normal cover of the complete bipartite graph $K_{4,4}$; if $s=4$, then $X$ is a normal cover of $B(P G(2,3))$ (the point-hyperplane incidence graph of the three-dimensional projective geometry $P G(2,3)$ ). As an application, we classify connected tetravalent $s$-transitive graphs of order $2 p^{2}$ for each prime $p$. It follows from this classification that, with the exception of $K_{4,4}$, all such graphs are 1-transitive. 
To end this section, we define a Cayley graph. Let $G$ be a permutation group on a set $\Omega$ and $\alpha \in \Omega$. Denote by $G_{\alpha}$ the stabilizer of $\alpha$ in $G$, that is, the subgroup of $G$ fixing the point $\alpha$. We say that $G$ is semiregular on $\Omega$ if $G_{\alpha}=1$ for every $\alpha \in \Omega$, and regular if $G$ is transitive and semiregular on $\Omega$. For a finite group $G$ and a subset $S$ of $G$ such that $1 \notin S$ and $S=S^{-1}$, the Cayley graph $\operatorname{Cay}(G, S)$ on $G$ with respect to $S$ is defined to have vertex set $G$ and edge set $\{\{g, s g\} \mid g \in G, s \in S\}$. Given $g \in G$, define the permutation $R(g)$ on $G$ by $x \mapsto x g$, for $x \in G$. The homomorphism $R$ is the right regular representation of $G$; the image $R(G)=\{R(g) \mid g \in G\}$ of $G$ is a regular permutation group acting on the elements of $G$. It is easy to see that $R(G)$ is isomorphic to $G$, which can therefore be regarded as a subgroup of the automorphism group $\operatorname{Aut}(\operatorname{Cay}(G, S))$. Thus the Cayley graph $\operatorname{Cay}(G, S)$ is vertextransitive. Furthermore, the group $\operatorname{Aut}(G, S)=\left\{\alpha \in \operatorname{Aut}(G) \mid S^{\alpha}=S\right\}$ is a subgroup of $\operatorname{Aut}(\operatorname{Cay}(G, S))_{1}$, the stabilizer of the vertex 1 in $\operatorname{Aut}(\operatorname{Cay}(G, S))$. A Cayley graph $\operatorname{Cay}(G, S)$ is said to be normal if $R(G)$ is normal in $\operatorname{Aut}(\operatorname{Cay}(G, S))$. Xu [28, Proposition 1.5] proved that $\operatorname{Cay}(G, S)$ is normal if and only if $\operatorname{Aut}(\operatorname{Cay}(G, S))_{1}=\operatorname{Aut}(G, S)$. A graph is called a circulant graph, or a circulant for short, if it is a Cayley graph on a cyclic group.

\section{Graph constructions and preliminaries}

In this section, we introduce some tetravalent $s$-transitive graphs of order twice a prime power, and collect some preliminary results which will be used later in the paper. Throughout this paper we denote by $\mathbb{Z}_{n}$ the cyclic group of order $n$ as well as the ring of integers modulo $n$, by $\mathbb{Z}_{n}^{*}$ the multiplicative group of $\mathbb{Z}_{n}$ consisting of numbers coprime to $n$, by $D_{2 n}$ the dihedral group of order $2 n$, and by $C_{n}, K_{n}$ and $n K_{1}$ the cycle, the complete graph and the null graph of order $n$, respectively. For two groups $M$ and $N, N \leq M$ means that $N$ is a subgroup of $M, N<M$ means that $N$ is a proper subgroup of $M, N \rtimes M$ denotes a semidirect product of $N$ by $M$, and $\operatorname{Aut}(N)$ denotes the automorphism group of $N$.

The first example is the lexicographic product of $C_{p^{2}}$ and $2 K_{1}$.

EXAMPLE 2.1. Let $p$ be a prime. The lexicographic product $C_{p^{2}}\left[2 K_{1}\right]$ is defined as the graph with vertex set $V\left(C_{p^{2}}\left[2 K_{1}\right]\right)=V\left(C_{p^{2}}\right) \times V\left(2 K_{1}\right)$ such that for any two vertices $u=\left(x_{1}, y_{1}\right)$ and $v=\left(x_{2}, y_{2}\right)$ in $V\left(C_{p^{2}}\left[2 K_{1}\right]\right), u$ is adjacent to $v$ in $C_{p^{2}}\left[2 K_{1}\right]$ whenever $\left\{x_{1}, x_{2}\right\} \in E\left(C_{p^{2}}\right)$.

Note that $C_{4}\left[2 K_{1}\right] \cong K_{4,4}$ is 3-transitive and $\operatorname{Aut}\left(C_{4}\left[2 K_{1}\right]\right) \cong\left(S_{4} \times S_{4}\right) \rtimes \mathbb{Z}_{2}$ if $p=2$, and the tetravalent graph $C_{p^{2}}\left[2 K_{1}\right]$ is 1-transitive and $\operatorname{Aut}\left(C_{p^{2}}\left[2 K_{1}\right]\right) \cong \mathbb{Z}_{2}^{p^{2}} \rtimes D_{2 p^{2}}$ if $p>2$. From [30, Table 1] we observe that $C_{p^{2}}\left[2 K_{1}\right]$ is isomorphic to $\operatorname{Cay}(G, S)$, where

$$
G=\langle a\rangle \times\langle b\rangle \cong \mathbb{Z}_{p^{2}} \times \mathbb{Z}_{2} \text { and } S=\left\{a, a^{-1}, a b, a^{-1} b\right\} .
$$

Next, we introduce some tetravalent symmetric Cayley graphs on an abelian group of order $2 p^{2}$ for prime $p$. 
EXAMPLE 2.2. Let $p$ be a prime congruent to $1 \bmod 4$, and let $H=\langle h\rangle$ be the unique subgroup of order 4 of $\mathbb{Z}_{2 p^{2}}^{*}$. Define $X_{2 p^{2}}^{0}=\operatorname{Cay}\left(G,\left\{a, a^{-1}, a^{h}, a^{h^{3}}\right\}\right)$, where $G=\langle a\rangle \cong \mathbb{Z}_{2 p^{2}}$.

$\mathrm{Xu}$ [29, Theorems 2 and 3] classified tetravalent symmetric circulant graphs, and one may deduce the following proposition.

Proposition 2.3. Let $p$ be a prime. A connected tetravalent circulant graph $X$ of order $2 p^{2}$ is symmetric and normal if and only if $p \equiv 1 \bmod 4$ and $X \cong X_{2 p^{2}}^{0}$. Furthermore, $\operatorname{Aut}\left(X_{2 p^{2}}^{0}\right) \cong \mathbb{Z}_{2 p^{2}} \rtimes \mathbb{Z}_{4}$.

EXAMPLE 2.4. Let $p$ be an odd prime and

$$
G=\langle a\rangle \times\langle b\rangle \times\langle c\rangle \cong \mathbb{Z}_{p} \times \mathbb{Z}_{p} \times \mathbb{Z}_{2} .
$$

Define $X_{2 p^{2}}^{1}=\operatorname{Cay}\left(G,\left\{c a, c a^{-1}, c b, c b^{-1}\right\}\right)$.

From [30, Theorem 3.3 and Proposition 3.3(iv)], one may deduce the following proposition.

PROPOSITION 2.5. Let $p$ be an odd prime, and

$$
G=\langle a\rangle \times\langle b\rangle \times\langle c\rangle \cong \mathbb{Z}_{p} \times \mathbb{Z}_{p} \times \mathbb{Z}_{2} .
$$

A connected tetravalent Cayley graph $X$ on $G$ is normal and symmetric if and only if $X \cong X_{2 p^{2}}^{1}$. Furthermore, $X_{2 p^{2}}^{1}$ is 1-transitive and $\operatorname{Aut}\left(X_{2 p^{2}}^{1}\right) \cong G \rtimes D_{8}$.

The following three infinite families of graphs were constructed by Gardiner and Praeger [14, Definitions 3.2, 4.2 and 4.3].

ExAmple 2.6. Let $p$ be an odd prime. The graph $\bar{X}_{2 p^{2}}^{1}$ is defined to have vertex set $\mathbb{Z}_{2} \times\left(\mathbb{Z}_{p} \times \mathbb{Z}_{p}\right)$ with two vertices $\left(0,\left(x_{1}, y_{1}\right)\right)$ and $\left(1,\left(x_{2}, y_{2}\right)\right)$ being adjacent if and only if

$$
\left(x_{2}, y_{2}\right)-\left(x_{1}, y_{1}\right) \in\{ \pm(1,0), \pm(0,1)\} .
$$

Note that the map defined by

$$
(i,(x, y)) \mapsto c^{i} a^{x} b^{y} \quad \forall(i,(x, y)) \in \mathbb{Z}_{2} \times\left(\mathbb{Z}_{p} \times \mathbb{Z}_{p}\right),
$$

is an isomorphism from $\bar{X}_{2 p^{2}}^{1}$ to $X_{2 p^{2}}^{1}$. Thus, $\bar{X}_{2 p^{2}}^{1} \cong X_{2 p^{2}}^{1}$.

EXAMPLE 2.7. Let $p$ be a prime congruent to $1 \bmod 4$, and let $\pm \varepsilon$ be the two elements of order 4 of $\mathbb{Z}_{p}^{*}$. The graph $X_{2 p^{2}}^{2}$ is defined to have vertex set $\mathbb{Z}_{2} \times\left(\mathbb{Z}_{p} \times \mathbb{Z}_{p}\right)$ with two vertices $\left(0,\left(x_{1}, y_{1}\right)\right)$ and $\left(1,\left(x_{2}, y_{2}\right)\right)$ being adjacent if and only if

$$
\left(x_{2}, y_{2}\right)-\left(x_{1}, y_{1}\right) \in\{(1,1),(-1, \varepsilon),(1,-1),(-1,-\varepsilon)\} .
$$


By [31, Lemma 3.1], $X_{2 p^{2}}^{2} \cong \operatorname{Cay}\left(G\left(2 p^{2}\right), S\right)$, where

$$
G\left(2 p^{2}\right)=\left\langle a, b, c \mid a^{p}=b^{p}=c^{2}=1, c a c=a^{-1}, c b c=b^{-1}, a b=b a\right\rangle
$$

is the generalized dihedral group of order $2 p^{2}$ and

$$
S=\left\{c a b, c a^{-1} b^{\varepsilon}, c a b^{-1}, c a^{-1} b^{-\varepsilon}\right\} .
$$

Furthermore, $\operatorname{Aut}\left(X_{2 p^{2}}^{2}\right) \cong G\left(2 p^{2}\right) \rtimes \mathbb{Z}_{4}$, implying that the tetravalent graph $X_{2 p^{2}}^{2}$ is 1-regular.

EXAMPLE 2.8. Let $p$ be an odd prime. The graph $X_{2 p^{3}}^{3}$ has vertex set $\mathbb{Z}_{2} \times \mathbb{Z}_{p}^{3}$, with two vertices $\left(0,\left(x_{0}, y_{0}, z_{0}\right)\right)$ and $\left(1,\left(x_{1}, y_{1}, z_{1}\right)\right)$ being adjacent if and only if

$$
\left(x_{1}, y_{1}, z_{1}\right)-\left(x_{0}, y_{0}, z_{0}\right) \in\{(1,0,0),(0,1,0),(0,0,1),(-1,-1,-1)\} .
$$

\section{LEMMA 2.9. Let $p$ be an odd prime, and let}

$$
\begin{gathered}
G\left(2 p^{3}\right)=\langle a, b, c, d| a^{p}=b^{p}=c^{p}=d^{2}=1, d a d=a^{-1}, d b d=b^{-1} \\
\left.d c d=c^{-1}, a b=b a, a c=c a, b c=c b\right\rangle
\end{gathered}
$$

be the generalized dihedral group of order $2 p^{3}$. Set $S=\left\{d a, d b, d c, d(a b c)^{-1}\right\}$. Then $X_{2 p^{3}}^{3} \cong \operatorname{Cay}\left(G\left(2 p^{3}\right), S\right)$. Also, $X_{2 p^{3}}^{3}$ is 2-transitive and $\operatorname{Aut}\left(X_{2 p^{3}}^{3}\right) \cong G\left(2 p^{3}\right) \rtimes S_{4}$.

Proof. Let $G=G\left(2 p^{3}\right), X=\operatorname{Cay}(G, S)$ and $A=\operatorname{Aut}(X)$. Note that $X_{2 p^{3}}^{3}$ has vertex set $\mathbb{Z}_{2} \times \mathbb{Z}_{p}^{3}$ with two vertices $\left(0,\left(x_{0}, y_{0}, z_{0}\right)\right)$ and $\left(1,\left(x_{1}, y_{1}, z_{1}\right)\right)$ adjacent whenever

$$
\left(x_{1}, y_{1}, z_{1}\right)-\left(x_{0}, y_{0}, z_{0}\right) \in\{(1,0,0),(0,1,0),(0,0,1),(-1,-1,-1)\} .
$$

It is easy to see that the map defined by

$$
(i,(x, y, z)) \mapsto d^{i} a^{x} b^{y} c^{z}, \quad \text { for }(i,(x, y, z)) \in \mathbb{Z}_{2} \times \mathbb{Z}_{p}^{3},
$$

is an isomorphism from $X_{2 p^{3}}^{3}$ to $X$. Thus, $X \cong X_{2 p^{3}}^{3}$.

Let $\alpha$ be the automorphism of $G$ induced by $a \mapsto b, b \mapsto c, c \mapsto(a b c)^{-1}, d \mapsto d$. Similarly, $\beta$ and $\gamma$ are the automorphisms of $G$ induced by $a \mapsto b, b \mapsto c, c \mapsto a$, $d \mapsto d$, and by $a \mapsto b, b \mapsto a, c \mapsto c, d \mapsto d$. It is easy to see that $\alpha, \beta$ and $\gamma$ fix $S$ setwise and hence they are automorphisms of $X$. Furthermore, $\alpha$ cyclicly permutes the elements in $S, \beta$ fixes $d(a b c)^{-1}$ and cyclicly permutes the remaining three elements in $S$, and $\gamma$ fixes $c$ and $d(a b c)^{-1}$ and interchanges $d a$ and $d b$. Since $S$ generates $G\left(2 p^{3}\right),\langle\alpha, \beta, \gamma\rangle$ acts faithfully on $S$. Thus, $\langle\alpha, \beta, \gamma\rangle \cong S_{4}$ and $R\left(G\left(2 p^{3}\right)\right) \rtimes\langle\alpha, \beta, \gamma\rangle$ is 2 -arc-transitive on $X$. If $p=3$, it can be shown with the 
help of the computer software package MAGMA [2] that $A=\operatorname{Aut}(X)$ has order 1296, which implies that $A=R\left(G\left(2 \cdot 3^{3}\right)\right) \rtimes\langle\alpha, \beta, \gamma\rangle$ and $X$ is 2-transitive. Let $p>3$. Then $X$ has girth 6 , and there are exactly two girth cycles passing through any given 2-arc. Thus, $X$ is not 3-arc-transitive because otherwise there are at least three girth cycles passing through any given 2-arc. Furthermore, $A_{1}^{*}=1$, where $A_{1}^{*}$ is the subgroup of $A_{1}$ fixing $S$ pointwise. As a result, $X$ is 2-transitive and $A=R\left(G\left(2 p^{3}\right)\right) \rtimes\langle\alpha, \beta, \gamma\rangle$.

The following proposition is taken from [14, Theorem 1.3].

PROPOSITION 2.10. Let $X$ be a connected tetravalent symmetric graph such that $\operatorname{Aut}(X)$ has an elementary abelian normal subgroup $N \cong \mathbb{Z}_{p}^{n}$, where $n>1$ and $p$ is an odd prime. If $N$ has exactly two orbits on vertices, then $X$ is isomorphic to $X_{2 p^{2}}^{1}$, $X_{2 p^{2}}^{2}$ or $X_{2 p^{3}}^{3}$.

EXAMPLE 2.11. Let $n$ be an integer greater than 2, and $q$ be a prime power. Let $P G(n-1, q)$ be the $(n-1)$-dimensional projective geometry over the field $G F(q)$. Denote by $P$ and $H$ the point set and the hyperplane set of $P G(n-1, q)$. The point-hyperplane incidence graph of $P G(n-1, q)$ is defined to have vertex set $P \cup H$ and edge set $\{\{x, y\} \mid x \in P, y \in H, x \in y\}$. The graph $B^{\prime}(P G(n-1, q))$ is defined to have vertex set $P \cup H$ and edge set $\{\{x, y\} \mid x \in P, y \in H, x \notin y\}$.

The following proposition is taken from [4, Theorem 2.4].

PROPOSITION 2.12. Let $p$ be a prime. A connected tetravalent symmetric graph of order $2 p$ is either 1-transitive or isomorphic to one of the following graphs.

\begin{tabular}{llll}
\hline$X$ & $|X|$ & $s$-transitive & $\operatorname{Aut}(X)$ \\
\hline$K_{5,5}-5 K_{2}$ & 10 & 2-transitive & $S_{5} \times \mathbb{Z}_{2}$ \\
$B^{\prime}(P G(2,2))$ & 14 & 2-transitive & $\operatorname{PGL}(3,2) .2$ \\
$B(P G(2,3))$ & 26 & 4-transitive & $\operatorname{PSL}(3,3) \rtimes \mathbb{Z}_{2}$ \\
\hline
\end{tabular}

Example 2.13. The 4-cube $Q_{4}$ may be viewed as the Cayley graph Cay $\left(\mathbb{Z}_{2}^{4}, S\right)$, where $\mathbb{Z}_{2}^{4}=\left\langle s_{1}\right\rangle \times\left\langle s_{2}\right\rangle \times\left\langle s_{3}\right\rangle \times\left\langle s_{4}\right\rangle$ and $S=\left\{s_{1}, s_{2}, s_{3}, s_{4}\right\}$.

The following proposition can be obtained from [18], where the connected 2-arctransitive graphs of order a prime power were characterized.

PROPOSITION 2.14. Let $X$ be a connected tetravalent s-transitive graph of order a prime power. Then $s=1,2$ or 3 . Furthermore, if $s=2$, then $X$ is a normal cover of the graphs $K_{4,4}, Q_{4}$ or $K_{5}$; if $s=3$, then $X$ is a normal cover of the complete bipartite $K_{4,4}$.

The following proposition is due to Praeger et al.; see [14, Theorem 1.1] and [24]. 
Proposition 2.15. Let $X$ be a connected tetravalent $(G, 1)$-arc-transitive graph. For each normal subgroup $N$ of $G$, one of the following holds:

(1) $N$ is transitive on $V(X)$;

(2) $X$ is bipartite and $N$ acts transitively on each part of the bipartition;

(3) $\quad N$ has $r \geq 3$ orbits on $V(X)$, the quotient graph $X_{N}$ is a cycle of length $r$, and $G$ induces the full automorphism group $D_{2 r}$ on $X_{N}$;

(4) $\quad N$ has $r \geq 5$ orbits on $V(X), N$ acts semiregularly on $V(X)$, the quotient graph $X_{N}$ is a connected tetravalent $G / N$-symmetric graph, and $X$ is a $G$-normal cover of $X_{N}$.

Moreover, if $X$ is also $(G, 2)$-arc-transitive, then case (3) cannot happen.

The next proposition characterizes the vertex stabilizer of the connected tetravalent $s$-transitive graphs, which can be deduced from [21, Lemma 2.5], [20, Proposition 2.8], or [19, Theorem 2.2].

Proposition 2.16. Let $X$ be a connected tetravalent $(G, s)$-transitive graph. Let $G_{v}$ be the stabilizer of a vertex $v \in V(X)$ in $G$. Then $s=1,2,3,4$ or 7 . Furthermore, either $G_{v}$ is a 2-group for $s=1$, or $G_{v}$ is isomorphic to $A_{4}$ or $S_{4}$ for $s=2 ; A_{4} \times \mathbb{Z}_{3}$, $\mathbb{Z}_{3} \times S_{4}, S_{3} \times S_{4}$ for $s=3 ; \mathbb{Z}_{3}^{2} \rtimes \operatorname{GL}(2,3)$ for $s=4$; or $\left[3^{5}\right] \rtimes \operatorname{GL}(2,3)$ for $s=7$, where $\left[3^{5}\right]$ represents an arbitrary group of order $3^{5}$.

\section{Main results}

In this section, we shall characterize connected tetravalent $s$-transitive graphs of order twice a prime power. To do this, we need the following lemma.

LEMMA 3.1. Let $p$ be a prime and let $n>1$ be an integer. Let $X$ be a connected tetravalent graph of order $2 p^{n}$. If $G \leq \operatorname{Aut}(X)$ is transitive on the arc set of $X$, then every minimal normal subgroup of $G$ is solvable.

Proof. Let $v \in V(X)$. Since $G$ is arc-transitive on $X$, by Proposition $2.16, G_{v}$ either is a 2-group or has order dividing $2^{4} \cdot 3^{6}$. It follows that $|G| \mid 2^{5} \cdot 3^{6} \cdot p^{n}$ or $|G|=2^{m+1} \cdot p^{n}$ for some integer $m$. Let $N$ be a minimal normal subgroup of $G$.

Suppose that $N$ is nonsolvable. Then $p>3$ and $|G| \mid 2^{5} \cdot 3^{6} \cdot p^{n}$ because a $\{2, p\}$ group is solvable by a theorem of Burnside [25, Theorem 8.5.3]. It follows that $X$ is $(G, 2)$-arc-transitive. Furthermore, 3||$N_{v} \mid$ for any $v \in V(X)$, because $p>3$, and the 2-arc-transitivity of $G$ implies that $N_{v}$ acts transitively on $X(v)$ because $N_{v} \unlhd G_{v}$. By Proposition 2.15, $N$ has at most two orbits on $V(X)$. Hence, $p^{n}$ divides $|N|$. Since $N$ is minimal, it is a product of isomorphic nonabelian simple groups. Since $|N| \mid 2^{5} \cdot 3^{6} \cdot p^{n}$, by [16, pp. 12-14], each direct factor of $N$ is one of the following:

$$
\begin{aligned}
& A_{5}, A_{6}, \operatorname{PSL}(2,7), \operatorname{PSL}(2,8), \operatorname{PSL}(2,17), \operatorname{PSL}(3,3), \operatorname{PSU}(3,3) \\
& \text { and } \operatorname{PSU}(4,2) .
\end{aligned}
$$

An inspection of the orders of such groups gives $n=2$ and $N \cong A_{5} \times A_{5}$. If $N$ is transitive on $V(X)$, then $X$ must be $(N, 2)$-transitive. Clearly, a direct factor $T$ of $N$ 
has at least $p=5$ orbits on $V(X)$. This forces $T$ to be semiregular on $V(X)$ which is impossible because $|V(X)|=2 p^{2}$. Thus, $N$ has exactly two orbits on $V(X)$. Then $N_{v} \cong A_{4} \times A_{4}$. This is also impossible by Proposition 2.14. Thus, $N$ is solvable.

THEOREM 3.2. Let $p$ be a prime and $s$ a positive integer. Let $X$ be a connected tetravalent $s$-transitive graph of order $2 p^{n}$. Then $s=1,2,3$ or 4 . Assume also that $s \geq 2$. Then $X$ is a normal cover of $Y$, where s and $Y$ are given in the following table.

\begin{tabular}{ll}
\hline$s$ & $Y$ \\
\hline 2 & $K_{4,4}, Q_{4}, K_{5}, K_{5,5}-5 K_{2}, B^{\prime}(P G(2,2))$ or $X_{2 p^{3}}^{3}$ \\
3 & $K_{4,4}$ \\
4 & $B(P G(2,3))$ \\
\hline
\end{tabular}

Proof. By Proposition 2.12, the theorem is true for $n=1$. Thus, one may assume that $n>1$ and $s>1$. Let $A=\operatorname{Aut}(X)$. For $p=2$, by Proposition 2.14, either $s=2$ and $X$ is a normal cover of the complete bipartite graph $K_{4,4}$ or the 4-cube $Q_{4}$, or $s=3$ and $X$ is a normal cover of $K_{4,4}$. Thus, assume $p>2$.

Let $M \unlhd A$ be maximal subject to $M$ having at least three orbits on $V(X)$. Since $X$ is 2-arc-transitive, by Proposition 2.15 , the quotient graph $X_{M}$ of $X$ relative to the orbit set of $M$ is a tetravalent $(A / M, s)$-transitive graph of order $2 p^{r}$ or $p^{t}$ with $1 \leq r, t \leq n$. Furthermore, $X$ is a normal cover of $X_{M}$. To complete the proof, it suffices to show that either $s=2$ and $X_{M} \cong K_{5}, K_{5,5}-5 K_{2}, B^{\prime}(P G(2,2))$ or $X_{2 p^{3}}^{3}$, or $s=4$ and $X_{M} \cong B(P G(2,3))$. Let $T / M$ be a minimal normal subgroup of $A / M$. Then $T / M$ has at most two orbits on $V\left(X_{M}\right)$.

Assume that $X_{M}$ has order $2 p^{r}$. Let $r>1$. By Lemma 3.1, $T / M \cong \mathbb{Z}_{p}^{r}$. Since $s>1$, by Proposition 2.10, $X_{M} \cong X_{2 p^{3}}^{3}$ and by Lemma 2.9, one has $s=2$. Now let $r=1$. By Proposition 2.12, $X_{M} \cong K_{5,5}-5 K_{2}, B^{\prime}(P G(2,2))$ or $B(P G(2,3))$. Clearly, if $X_{M} \cong K_{5,5}-5 K_{2}$ or $B^{\prime}(P G(2,2))$, then $X_{M}$ is 2-transitive and hence $X$ is 2transitive. If $X_{M} \cong B(P G(2,3))$, then $p=13$ and $X_{M}$ is 4-transitive, implying that $X$ is at most 4-transitive. Note that $A / M \leq \operatorname{Aut}\left(X_{M}\right)$. For a vertex $\bar{v} \in V\left(X_{M}\right)$, let $(A / M)_{\bar{v}}$ and $\operatorname{Aut}\left(X_{M}\right)_{\bar{v}}$ be the stabilizers of $\bar{v}$ in $A / M$ and $\operatorname{Aut}\left(X_{M}\right)$, respectively. Then $(A / M)_{\bar{v}} \leq \operatorname{Aut}\left(X_{M}\right)_{\bar{v}}$ and $\operatorname{Aut}\left(X_{M}\right)_{\bar{v}} \cong \mathbb{Z}_{3}^{2} \rtimes \operatorname{GL}(2,3)$ by Proposition 2.16. Suppose that $(A / M)_{\bar{v}} \neq \operatorname{Aut}\left(X_{M}\right)_{\bar{v}}$. Then $X_{M}$ is $(A / M, s)$-transitive for $s=2$ or 3. Again by Proposition 2.16, $(A / M)_{\bar{v}}$ has a subgroup isomorphic to $A_{4}$. However, $\mathbb{Z}_{3}^{2} \rtimes \mathrm{GL}(2,3)$ has no subgroups isomorphic to $A_{4}$, a contradiction. Thus, $(A / M)_{\bar{v}}=\operatorname{Aut}\left(X_{M}\right)_{\bar{v}}$, and hence $X$ is 4-transitive.

Now assume that $X_{M}$ has order $p^{t}$. By Proposition 2.14, $X_{M}$ is a normal cover of the complete graph $K_{5}$. This means that $p=5$, and there exists a normal 5-subgroup, say $\bar{H}$, in $\operatorname{Aut}\left(X_{M}\right)$ such that the quotient graph $\left(X_{M}\right)_{\bar{H}}$ of $X_{M}$ relative to the orbit set of $\bar{H}$ is $K_{5}$. Further, $\operatorname{Aut}\left(X_{M}\right) / \bar{H} \leq \operatorname{Aut}\left(K_{5}\right) \cong S_{5}$. Noting that $\operatorname{Aut}\left(X_{M}\right) / \bar{H}$ is 
TABLE 1. Tetravalent $s$-transitive graphs of order $2 p^{2}$.

\begin{tabular}{llll}
\hline$X$ & $s$-transitive & $\operatorname{Aut}(X)$ & Comments \\
\hline$K_{4,4}$ & 3-transitive & $\left(S_{4} \times S_{4}\right) \rtimes \mathbb{Z}_{2}$ & Example 2.1, $p=2$ \\
$C_{p^{2}}\left[2 K_{1}\right]$ & 1-transitive & $\mathbb{Z}_{2}^{p^{2} \rtimes D_{2 p^{2}}}$ & Example 2.1, $p>2$ \\
$X_{2 p^{2}}^{0}$ & 1-regular & $\mathbb{Z}_{2 p^{2} \rtimes \mathbb{Z}_{4}}$ & Example 2.2, $p \equiv 1 \bmod 4$ \\
$X_{2 p^{2}}^{1}$ & 1-transitive & $\left(\mathbb{Z}_{2} \times \mathbb{Z}_{p}^{2}\right) \rtimes D_{8}$ & Example 2.4, $p>2$ \\
$X_{2 p^{2}}^{2}$ & 1-regular & $G\left(2 p^{2}\right) \rtimes \mathbb{Z}_{4}$ & Example 2.7, $p \equiv 1 \bmod 4$ \\
\hline
\end{tabular}

2-arc-transitive on $K_{5}$, one has $\operatorname{Aut}\left(X_{M}\right) / \bar{H} \cong A_{5}$ or $S_{5}$. This tells us that $\bar{H}$ is the largest normal 5-subgroup of $\operatorname{Aut}\left(X_{M}\right)$. It is easily seen that $A / M \leq \operatorname{Aut}\left(X_{M}\right)$ and that every Sylow 5-subgroup of $A / M$ is also a Sylow 5-subgroup of $\operatorname{Aut}\left(X_{M}\right)$. Therefore, $\bar{H}$ is also a normal subgroup of $A / M$. Set $H / M=\bar{H}$. Then $H \unlhd A$, and it is easy to see that $H$ has five orbits on $V(X)$. The maximality of $M$ forces that $H=M$. Thus, $t=1$ and $X_{M} \cong K_{5}$. In particular, $s=2$.

Let $G$ be a nonabelian simple group and $Z$ an abelian group. We call an extension $E$ of $Z$ by $G$ a central extension of $G$ if $Z \leq Z(E)$. If $E$ is perfect, that is, the derived group $E^{\prime}=E$, we call $E$ a covering group of $G$. Schur proved that for every simple group $G$ there is a unique maximal covering group $M$ such that every covering group of $G$ is a factor group of $M$. This group $M$ is called the full covering group of $G$, and the center of $M$ is called the Schur multiplier of $G$, denoted by $\operatorname{Mult}(G)$. For more information for the Schur multiplier, see, for example, [17, Ch. 5].

THEOREM 3.3. Let $p$ be a prime and $X$ a connected tetravalent graph of order $2 p^{2}$. Then $X$ is s-transitive for some positive integer s if and only if it is isomorphic to one of the graphs in Table 1. Furthermore, all graphs in Table 1 are pairwise nonisomorphic.

PROOF. By Examples 2.1-2.7, all graphs in Table 1 are pairwise nonisomorphic tetravalent symmetric graphs. Let $X$ be a tetravalent $s$-transitive graph of order $2 p^{2}$ for an integer $s$. To finish the proof, it suffices to show that $X$ is one of the graphs listed in Table 1. If $p \leq 3$ then $|X|=8$ or 18 , and by [22], up to isomorphism there are three connected tetravalent symmetric graph of order 8 or 18 . It follows that $X \cong K_{4,4}, C_{9}\left[2 K_{1}\right]$ or $X_{2 \cdot 3^{2}}^{1}$. In what follows, we assume that $p>3$. Set $A=\operatorname{Aut}(X)$ and let $v \in V(X)$. We first prove a claim.

Claim. If $A$ has a nontrivial normal 2-subgroup, say $M$, then $X$ is isomorphic to $C_{p^{2}}\left[2 K_{1}\right], X_{2 p^{2}}^{0}$ or $X_{2 p^{2}}^{1}$.

Consider the quotient graph $X_{M}$ of $X$ relative to the orbit set of $M$, and let $K$ be the kernel of $A$ acting on $V\left(X_{M}\right)$. Since $p>3$, every orbit of $M$ has length 2, and 
hence $\left|X_{M}\right|=p^{2}$. By the symmetry of $X$, every orbit of $M$ contains no edges, and by Proposition $2.15, X_{M}$ is of valency 2 or 4 . If $X_{M}$ has valency 2 , then $X \cong C_{p^{2}}\left[2 K_{1}\right]$. If $X_{M}$ has valency 4 , by Proposition $2.15, K$ is semiregular, implying that $K=M \cong \mathbb{Z}_{2}$. Let $P$ be a Sylow $p$-subgroup of $A$. Then $|P|=p^{2}$ and hence $P$ is abelian. Since $X_{M}$ is a tetravalent graph of order $p^{2}$ and $p>2, P M / M$ must be regular on $V\left(X_{M}\right)$. It follows that $X_{M}$ is a Cayley graph on $P M / M$. By [1, Corollary 1.3], every connected tetravalent Cayley graph on an odd order abelian group $G$ is normal except for $G=\mathbb{Z}_{5}$. It follows that $X_{M}$, as a Cayley graph on $P M / M$, is normal, and hence $P M / M \unlhd A / M$, namely, $P M \unlhd A$. It is easily seen that $P M$ is transitive on $V(X)$. Since $|P M|=2 p^{2}$, $P M$ is also regular on $V(X)$, implying that $X$ is a normal Cayley graph on $P M$. Since $M$ is a normal subgroup of order $2, M$ is in the center of $A$, implying that $P M$ is abelian. By Propositions 2.3 and $2.5, X \cong X_{2 p^{2}}^{0}$ or $X_{2 p^{2}}^{1}$, as claimed.

Now take a minimal normal subgroup, say $N$, in $A$. By Lemma $3.1, N$ is solvable. Since $p>3$ and $|V(X)|=2 p^{2}, N$ is an elementary abelian 2-group or $p$ group. By the claim, we may assume further that $N$ is a $p$-group. Take a nontrivial maximal normal $p$-subgroup, say $M$, of $A$. Clearly, since $p>3$, one has $|M| \mid p^{2}$. If $M \cong \mathbb{Z}_{p} \times \mathbb{Z}_{p}$, then $M$ has exactly two orbits on $V(X)$, and by Proposition $2.10, X \cong X_{2 p^{2}}^{1}$ or $X_{2 p^{2}}^{2}$. Assume now that $M$ is cyclic. Set $C=C_{A}(M)$. Then $M \leq C$ and $A / C \leq \operatorname{Aut}(M) \cong \mathbb{Z}_{p(p-1)}$.

Suppose that $C=M$. Then $M \cong \mathbb{Z}_{p^{2}}$. Clearly, $M$ acts semiregularly on $V(X)$ with two orbits. Let $R(M)$ and $L(M)$ be the two orbits of $M$. Since $M$ acts regularly on $R(M)$ and $L(M)$, one may assume that $R(M)=\{R(g) \mid g \in M\}$ and $L(M)=\{L(g) \mid g \in M\}$, and that the actions of $M$ on $R(M)$ and $L(M)$ are just by right multiplication, that is, $R(h)^{g}=R(h g)$ and $L(h)^{g}=L(h g)$ for any $h, g \in M$. By the symmetry of $X$, there is no edge in $R(M)$ or $L(M)$, implying that $X$ is bipartite. Let the neighbors of $R(1)$ be $L\left(g_{1}\right), L\left(g_{2}\right), L\left(g_{3}\right)$ and $L\left(g_{4}\right)$, where $g_{1}, g_{2}, g_{3}, g_{4} \in M$. Since $M$ is abelian, for any $g \in M$, the neighbors of $R(g)$ are $L\left(g g_{1}\right), L\left(g g_{2}\right), L\left(g g_{3}\right)$ and $L\left(g g_{4}\right)$, and furthermore, the neighbors of $L(g)$ are $R\left(g g_{1}^{-1}\right), R\left(g g_{2}^{-1}\right), R\left(g g_{3}^{-1}\right)$ and $R\left(g g_{4}^{-1}\right)$. The map $\alpha$ defined by $R(g) \mapsto L\left(g^{-1}\right), L(g) \mapsto R\left(g^{-1}\right)$ for any $g \in G$, is an automorphism of $X$ of order 2. Let $B=\langle M, \alpha\rangle$. Then $B$ is transitive on $V(X)$, and since $M$ is normal in $A, B=M \rtimes\langle\alpha\rangle$ has order $2 p^{2}$, and hence it acts regularly on $V(X)$. Thus, $A=B A_{v}$, where $A_{v}$ is the stabilizer of $v \in V(X)$ in $A$. Since $|M|=p^{2}, A_{v} M / M$ has even order and since $B / M$ has order $2, A / M$ is not cyclic. This leads to a contradiction.

Thus, $M<C$. Let $T / M$ be a minimal normal subgroup of $A / M$ contained in $C / M$. Suppose that $T / M$ is nonsolvable. Then $M \cong \mathbb{Z}_{p}$ and $X$ is 2 -arc-transitive. It follows that the quotient graph $X_{M}$ of $X$ relative to the orbit set of $M$ is a tetravalent 2 -arc-transitive graph of order $2 p$. From Proposition 2.12 it can be easily deduced that $T / M \cong A_{5}, \operatorname{PSL}(2,7)$ or $\operatorname{PSL}(3,3)$. Let $T^{\prime}$ be the derived subgroup of $T$. Since $T / M$ is simple, one has $T^{\prime} M / M=T / M$, that is, $T=T^{\prime} M$. If $M \leq T^{\prime}$, then $T^{\prime}=T$, and $T$ is a covering group of one of the groups $A_{5}, \operatorname{PSL}(2,7)$ and $\operatorname{PSL}(3,3)$. However, by [6], Mult $\left(A_{5}\right) \cong \operatorname{Mult}(\operatorname{PSL}(2,7)) \cong \mathbb{Z}_{2}$ and $\operatorname{Mult}(\operatorname{PSL}(3,3))=1$. This 
forces $p \leq 2$, a contradiction. Thus, $M \not T^{\prime}$. Since $M \cong \mathbb{Z}_{p}, T^{\prime} \cap M=1$ and $T=T^{\prime} \times M$. Clearly, $T^{\prime}$ is nonabelian simple. Then $T^{\prime}$ is characteristic in $T$, and hence it is normal in $A$ because $T \unlhd A$, contrary to Lemma 3.1. Thus, $T / M$ is solvable. Then $T / M$ must be a 2-group and hence $T=M \times Q$, where $Q$ is a Sylow 2-subgroup of $T$. Then $Q$ is characteristic in $T$, and since $T \unlhd A$, one has $Q \unlhd A$. By the claim, $X \cong C_{p^{2}}\left[2 K_{1}\right], X_{2 p^{2}}^{0}$ or $X_{2 p^{2}}^{1}$.

COROLlary 3.4. Let $p$ be an odd prime. Every connected tetravalent symmetric graph of order $2 p^{2}$ is a 1-transitive Cayley graph.

\section{Acknowledgement}

The authors are indebted to the referee for many valuable comments and constructive suggestions.

\section{References}

[1] Y. G. Baik, Y.-Q. Feng, H. S. Sim and M. Y. Xu, 'On the normality of Cayley graphs of abelian groups', Algebra Colloq. 5 (1998), 297-304.

[2] W. Bosma, C. Cannon and C. Playoust, 'The MAGMA algebra system I: The user language', J. Symbolic Comput. 24 (1997), 235-265.

[3] C. Y. Chao, 'On the classification of symmetric graphs with a prime number of vertices', Trans. Amer. Math. Soc. 158 (1971), 247-256.

[4] Y. Cheng and J. Oxley, 'On weakly symmetric graphs of order twice a prime', J. Combin. Theory Ser. B 42 (1987), 196-211.

[5] M. D. E. Conder and C. E. Praeger, 'Remarks on path-transitivity on finite graphs', European J. Combin. 17 (1996), 371-378.

[6] J. H. Conway, R. T. Curties, S. P. Norton, R. A. Parker and R. A. Wilson, An Atlas of Finite Groups (Clarendon Press, Oxford, 1985).

[7] D. Ž. Djoković and G. L. Miller, 'Regular groups of automorphisms of cubic graphs', J. Combin. Theory Ser. B 29 (1980), 195-230.

[8] X. G. Fang, C. H. Li and M. Y. Xu, 'On edge-transitive Cayley graphs of valency four', European J. Combin. 25 (2004), 1107-1116.

[9] Y.-Q. Feng and J. H. Kwak, 'One-regular cubic graphs of order a small number times a prime or a prime square', J. Aust. Math. Soc. 76 (2004), 345-356.

[10] Y.-Q. Feng and J. H. Kwak, 'Classifying cubic symmetric graphs of order $10 p$ or $10 p^{2}$, Sci. China Ser. A 49 (2006), 300-319.

[11] Y.-Q. Feng and J. H. Kwak, 'Cubic symmetric graphs of order twice an odd prime power', J. Aust. Math. Soc. 81 (2006), 153-164.

[12] Y.-Q. Feng and J. H. Kwak, 'Cubic symmetric graphs of order a small number times a prime or a prime square', J. Combin. Theory Ser. B 97 (2007), 627-646.

[13] Y.-Q. Feng, J. H. Kwak and K. S. Wang, 'Classifying cubic symmetric graphs of order $8 p$ or $8 p^{2}$, , European J. Combin. 26 (2005), 1033-1052.

[14] A. Gardiner and C. E. Praeger, 'On 4-valent symmetric graphs', European J. Combin. 15 (1994), 375-381.

[15] A. Gardiner and C. E. Praeger, 'A characterization of certain families of 4-valent symmetric graphs', European. J. Combin. 15 (1994), 383-397.

[16] D. Gorenstein, Finite Simple Groups (Plenum Press, New York, 1982).

[17] B. Huppert, Endliche Gruppen I (Springer, Berlin, 1967). 
[18] C. H. Li, 'Finite $s$-arc-transitive graphs of prime-power order', Bull. Lond. Math. Soc. 33 (2001), 129-137.

[19] C. H. Li, 'The finite vertex-primitive and vertex-biprimitive $s$-transitive graphs for $s \geq 4$ ', Trans. Amer. Math. Soc. 353 (2001), 3511-3529.

[20] C. H. Li, Z. P. Lu and D. Marušič, 'On primitive permutation groups with small suborbits and their orbital graphs', J. Algebra 279 (2004), 749-770.

[21] C. H. Li, Z. P. Lu and H. Zhang, 'Tetravalent edge-transitive Cayley graphs with odd number of vertices', J. Combin. Theory Ser. B 96 (2006), 164-181.

[22] B. D. McKay, 'Transitive graphs with fewer than 20 vertices', Math. Comp. 33 (1979), 1101-1121.

[23] R. C. Miller, 'The trivalent symmetric graphs of girth at most six', J. Combin. Theory Ser. B 10 (1971), 163-182.

[24] C. E. Praeger, 'An O'Nan-Scott theorem for finite quasiprimitive permutation groups and an application to 2-arc transitive graphs', J. Lond. Math. Soc. 47 (1992), 227-239.

[25] D. J. S. Robinson, A Course in the Theory of Groups (Springer, New York, 1982).

[26] W. T. Tutte, 'A family of cubical graphs', Proc. Camb. Phil. Soc. 43 (1947), 621-624.

[27] R. J. Wang and M. Y. Xu, 'A classification of symmetric graphs of order $3 p$ ', J. Combin. Theory Ser. B 58 (1993), 197-216.

[28] M. Y. Xu, 'Automorphism groups and isomorphisms of Cayley digraphs', Discrete Math. 182 (1998), 309-319.

[29] M. Y. Xu, 'A note on one-regular graphs', Chinese Sci. Bull. 45 (2000), 2160-2162.

[30] J. Xu and M. Y. Xu, 'Arc-transitive Cayley graphs of valency at most four on abelian groups', Southeast Asian Bull. Math. 25 (2001), 355-363.

[31] J.-X. Zhou and Y.-Q. Feng, 'Tetravalent one-regular graphs of order 2pq', J. Algebraic Combin. 29 (2009), 457-471.

JIN-XIN ZHOU, Department of Mathematics, Beijing Jiaotong University, Beijing 100044, PR China

e-mail: 05118322@bjtu.edu.cn, jxzhou@bjtu.edu.cn

YAN-QUAN FENG, Department of Mathematics, Beijing Jiaotong University, Beijing 100044, PR China

e-mail: yqfeng@bjtu.edu.cn 